BMJ Open Diabetes Research \& Care

\section{Design of and rationale for the Japan Diabetes Optimal Integrated Treatment study for 3 major risk factors of cardiovascular diseases (J-DOIT3): a multicenter, open-label, randomized, parallel-group trial}

To cite: Ueki K, Sasako T, Kato $\mathrm{M}$, et al. Design of and rationale for the Japan Diabetes Optimal Integrated Treatment study for 3 major risk factors of cardiovascular diseases (J-DOIT3): a multicenter, open-label, randomized, parallel-group trial. BMJ Open Diabetes Research and Care 2016;4: e000123. doi:10.1136/ bmjdrc-2015-000123

- Additional material is available. To view please visit the journal (http://dx.doi.org/ 10.1136/bmjdrc-2015000123)

Received 10 June 2015 Revised 22 October 2015 Accepted 26 October 2015

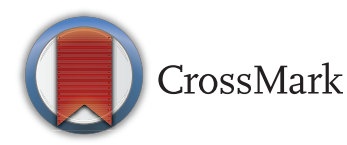

For numbered affiliations see end of article.

Correspondence to Dr Takashi Kadowaki; kadowaki-3im@h.u-tokyo.ac.jp

\section{ABSTRACT}

Objective: Multifactorial intervention including the management of levels of blood glucose (BG), blood pressure (BP), and lipids has been suggested to decrease cardiovascular disease (CVD) risk. However, the target ideal and feasible levels for these individual parameters have not been fully evaluated. In this study, we examine the hypothesis that stricter control compared with the current targets in the Japanese guideline for BG, BP, and lipids could efficiently and safely reduce CVD risk.

Research Design and Methods: We screened patients with type 2 diabetes and hypertension and/or dyslipidemia among 81 hospitals in Japan and allocated them into 2 groups: the intensive therapy group (ITG) and the conventional therapy group (CTG). For the 2 respective groups, the target for glycated hemoglobin $(\mathrm{HbA} 1 \mathrm{c})$ is $<6.2 \%$ $(44 \mathrm{mmol} / \mathrm{mol})$ and $<6.9 \%(52 \mathrm{mmol} / \mathrm{mol})$, for BP it is $<120 / 75 \mathrm{~mm} \mathrm{Hg}$ and $<130 / 80 \mathrm{~mm} \mathrm{Hg}$, and for low-density lipoprotein cholesterol it is $<80 \mathrm{mg} / \mathrm{dL}$ $(<70 \mathrm{mg} / \mathrm{dL}$ in the presence of CVD history) and $<120 \mathrm{mg} / \mathrm{dL}(<100 \mathrm{mg} / \mathrm{dL}$ in the presence of CVD history). The primary end point is the occurrence of CVD events or death by any cause. These patients are scheduled for stepwise intensifications of medication for BG, BP, and lipid control in the ITG, until the number of primary end point events reaches 250 .

Results: We recruited 2542 patients and randomly allocated 1271 into the ITG and 1271 into the CTG between June 2006 and March 2009. The mean HbA1c was $8.0 \%(64 \mathrm{mmol} / \mathrm{mol})$ and the mean duration of diabetes was 8.3 years.

Conclusions: This randomized controlled study will test the hypothesis that strict multifactorial intervention therapy is effective for the prevention of CVDs in patients with type 2 diabetes who are at high CVD risk. Trial registration number: NCT00300976.

\section{Key messages}

- We successfully set up a randomized control study to test the hypothesis that strict multifactorial intervention therapy is effective for the prevention of cardiovascular diseases in patients of type 2 diabetes mellitus at high risk.

- We recruited 2542 patients in 81 facilities in Japan and randomly allocated 1271 patients into the intensive therapy group.

- This study continues until the number of primary end point events, including death, myocardial infarction, and stroke, reaches 250 .

\section{INTRODUCTION}

The epidemic of type 2 diabetes mellitus is a serious health issue worldwide. In Japan, 9.5 million people are estimated to have this disease (National Health Surveillance 2013) that is characterized by chronic hyperglycemia associated with insufficient insulin action that leads to disorders in glucose, lipid, and protein metabolism. When these metabolic disorders are chronic, they can manifest diabetic microvascular and macrovascular complications. Macrovascular lesions involving large vessels, such as the coronary artery, cerebral artery, or arteries of the lower extremities, account for myocardial infarction, cerebral infarction, and peripheral artery disease, whereas microvascular lesions account for visual impairment, renal failure, and neuropathy. Epidemiological studies, including the Hisayama study, demonstrate that diabetes is associated with a near threefold risk for the onset of macrovascular 
diseases such as myocardial infarction and stroke, and that this risk becomes greater when it is accompanied by hypertension and/or dyslipidemia. ${ }^{1}$ Furthermore, a total of 16000 patients are estimated to start receiving hemodialysis annually due to diabetic nephropathy, and a large number of patients develop ischemic heart disease or stroke, at an incidence of 17.0/1000 person-years in the Japan Diabetes Complications Study (JDCS). ${ }^{2}$ No evidence has been obtained, however, that glycemic control will prevent cardiovascular diseases (CVDs) in patients with diabetes in the Japanese population, although the Kumamoto study showed that intensive insulin therapy reduced the risk of microvascular complications. ${ }^{3}$

Globally, a number of clinical studies evaluating various antidiabetic therapeutic strategies have been conducted to provide evidence that intensive therapy reduces microvascular and macrovascular complications. Of these, the UK Prospective Diabetes Study 33 (UKPDS 33 ), in which a total of 3867 patients with type 2 diabetes were randomly assigned to intensive therapy with sulfonylurea/insulin or to conventional diet therapy for glycemic control, came to a noteworthy conclusion that intensive therapy led to a decrease in the risk for the onset of microvascular complications among the treated patients, compared with diet therapy alone. ${ }^{4}$ Moreover, in the UKPDS 35, in which the results of the UKPDS were further analyzed epidemiologically, it was shown that decreases in glycated hemoglobin (HbAlc) are paralleled with decreases in the risk for mortality, myocardial infarction, and microvascular complications. ${ }^{5}$ Furthermore, the 10-year post-trial observational study of UKPDS revealed the existence of a 'legacy effect' that caused intensive blood glucose (BG) control immediately after disease onset to be associated with long-term beneficial effects on the prevention of coronary events and death by any cause. ${ }^{6}$ For the patients with advanced type 2 diabetes, the PROactive (PROspective pioglitAzone Clinical Trial In macroVascular Events) study demonstrated that pioglitazone added to conventional therapy produced greater reductions in the onset of macrovascular complications than did placebo, indicating that intensive therapy with pioglitazone is efficacious for the secondary prevention of macrovascular complications. $^{7}$ However, recent trials challenging whether controlling hyperglycemia to near normal levels of HbAlc could reduce the risk of macrovascular complications, such as the ACCORD (Action to Control Cardiovascular Risk in Diabetes) trial, ${ }^{8}$ the ADVANCE (Action in Diabetes and Vascular Disease: PreterAx and DiamicroN Modified Release Controlled Evaluation) trial, ${ }^{9}$ and the VADT (Veterans Affairs Diabetes Trial), ${ }^{10}$ failed to show the beneficial effect of intensive BG control for patients with advanced stages of diabetes, presumably due to a marked increase in severe hypoglycemic episodes.

However, the Steno-2 study, in which patients with type 2 diabetes with microalbuminuria were randomly assigned to intensive therapy for rigorous BG, blood pressure (BP), and lipid control or to conventional therapy, reported a reduction in the incidence of microvascular and macrovascular complications in patients treated with multifactorial intensive therapy. ${ }^{11}$ Moreover, the 5-year post-trial observational study of Steno-2 showed a significant reduction in macrovascular complications and mortality in the intensive therapy group (ITG), ${ }^{12}$ suggesting that the comprehensive and strict control of BG, BP, and lipids brings with it long-term beneficial effects on the prevention of macrovascular complications and death, due to the possible 'legacy effect'. Still, the study had limitations, including the small number of participants, only 160 . In addition, glycemic control may not have been sufficient, because the mean HbAlc was nearly $8 \%(64 \mathrm{mmol} / \mathrm{mol})$ and the achievement rate of the target $\mathrm{HbAlc},<6.5 \%$ $(48 \mathrm{mmol} / \mathrm{mol})$, was around $15 \%$, even in the ITG.

Effectiveness of multifactorial intervention was investigated also in the ADDITION-Europe (Anglo-DanishDutch Study of Intensive Treatment In People with Screen Detected Diabetes in Primary Care), in which over 3000 patients with newly diagnosed type 2 diabetes were recruited, whose mean baseline HbAlc was about $7 \%(53 \mathrm{mmol} / \mathrm{mol})$, resulting in a slight but nonsignificant reduction in CVD events. ${ }^{13}$

Most recently, the EMPA-REG OUTCOME study showed that a sodium-glucose linked co-transporter 2 (SGLT2) inhibitor, empagliflozin, reduced cardiovascular mortality, with no difference in incidence of myocardial infarction or stroke. ${ }^{14}$

In 2005, the Ministry of Health, Labor and Welfare (MHLW) of Japan developed a new category of research projects, namely 'strategic research', whose target diseases were of the highest social priority and whose results were expected to be immediately applied to the clinical practice and the Japanese healthcare system. In the field of diabetes, three trials were planned; specifically, the Japan Diabetes Outcome Intervention Trial $(\mathrm{J}$-DOIT $),{ }^{15}$ including J-DOIT1, aimed at reducing the conversion from impaired glucose tolerance to diabetes through education and lifestyle modification; ${ }^{16}$ J-DOIT2 aimed at reducing patient dropout rates from diabetes treatment through improved therapy from primary care physicians $;{ }^{17}$ and J-DOIT3 aimed at reducing macrovascular complications through multifactorial interventions. Here, we describe the design of and rationale for J-DOIT3, renamed the Japan Diabetes Optimal Integrated Treatment study for three major risk factors of cardiovascular diseases, which started in 2006 and is, as of this writing, still underway.

\section{RESEARCH DESIGN AND METHODS Study design}

The present trial is a multicenter, open-label, randomized, parallel-group study, conducted to examine the efficacy of intensive multifactorial therapy on the 
development and exacerbation of diabetic complications, in particular CVDs, and mortality in patients with type 2 diabetes with high risk for CVDs, whose details are available online. ${ }^{18}$ In this trial, patients with type 2 diabetes who were at high risk of macrovascular complications were recruited, allocated to either the conventional therapy group (CTG) or the ITG, and followed up (figure 1).

\section{Organization and funding}

Research funding is to be accounted for by the MHLW grants in aid, as well as corporate donations, as described later, and to be supplied through the funding agency, which was shifted from the Japan Foundation for the Promotion of International Medical Research Co-operation (the National Center for Global Health and Medicine, Tokyo, Japan), or JF-PIMRC, to the Japan Diabetes Foundation (JDF) in April 2013 (see sFigure 1).

To monitor and assess the research, the foundation organized the Trial Coordinating Committee, the Trial Administrative Committee, the Trial Assessment Committee, the Trial Progress Management Committee, the Safety Assessment Committee, the Central Ethics Committee, and the Endpoint Assessment Committees, whose purposes are referred to online. ${ }^{18}$ Among them, the Endpoint Assessment Committees are blinded to the study arm, whereas the Trial Assessment Committee and the Safety Assessment Committee are not. The protocol of this study, as further mentioned later, was approved by the Central Ethics Committee.

\section{Eligibility}

Medical institutions were selected in Japan that employed certified diabetologist(s) who were expected to register 50 or more participants (25 or more participants per group) with type 2 diabetes and hypertension and/or dyslipidemia. The inclusion and exclusion criteria, shown in sTable 1 and 2, respectively, were formulated to recruit those patients who were at high risk of CVDs, but still allow for intensification of medication for BG, BP, and lipids.

\section{Informed consent, registration, and random allocation}

The physician in charge explained the study particulars in person to each candidate participant to obtain consent to participate in the study, and the written document of informed consent was obtained one or more days after the date of the physician's explanation.

On receiving the patient's registration, the Data Center was to confirm the eligibility of the participant, followed by dynamic allocation to the CTG or to the ITG in a 1:1 ratio through randomization adjusted by the presence or absence of CVD (myocardial infarction, stroke, coronary bypass surgery, percutaneous coronary angioplasty, cerebral revascularization, or cerebrovascular reconstruction), male/female ratio, age $(\geq 60$, $<60$ years old), and HbAlc $(\geq 8.9 \% \quad(74 \mathrm{mmol} / \mathrm{mol})$, $<8.9 \%$ ), to ensure a balanced allocation of participant characteristics between the groups (figure 1).

After registration, each time the protocol is revised, informed consent is reobtained in all participants receiving investigational treatment. For participants who are
Figure 1 Screening, enrollment, and randomization of study participants (BMI, body mass index; CTG, conventional therapy group; HbA1c, glycated hemoglobin; HDL-C, high-density lipoprotein cholesterol; IHD, ischemic heart disease; ITG, intensive therapy group; LDL-C, low-density lipoprotein cholesterol).

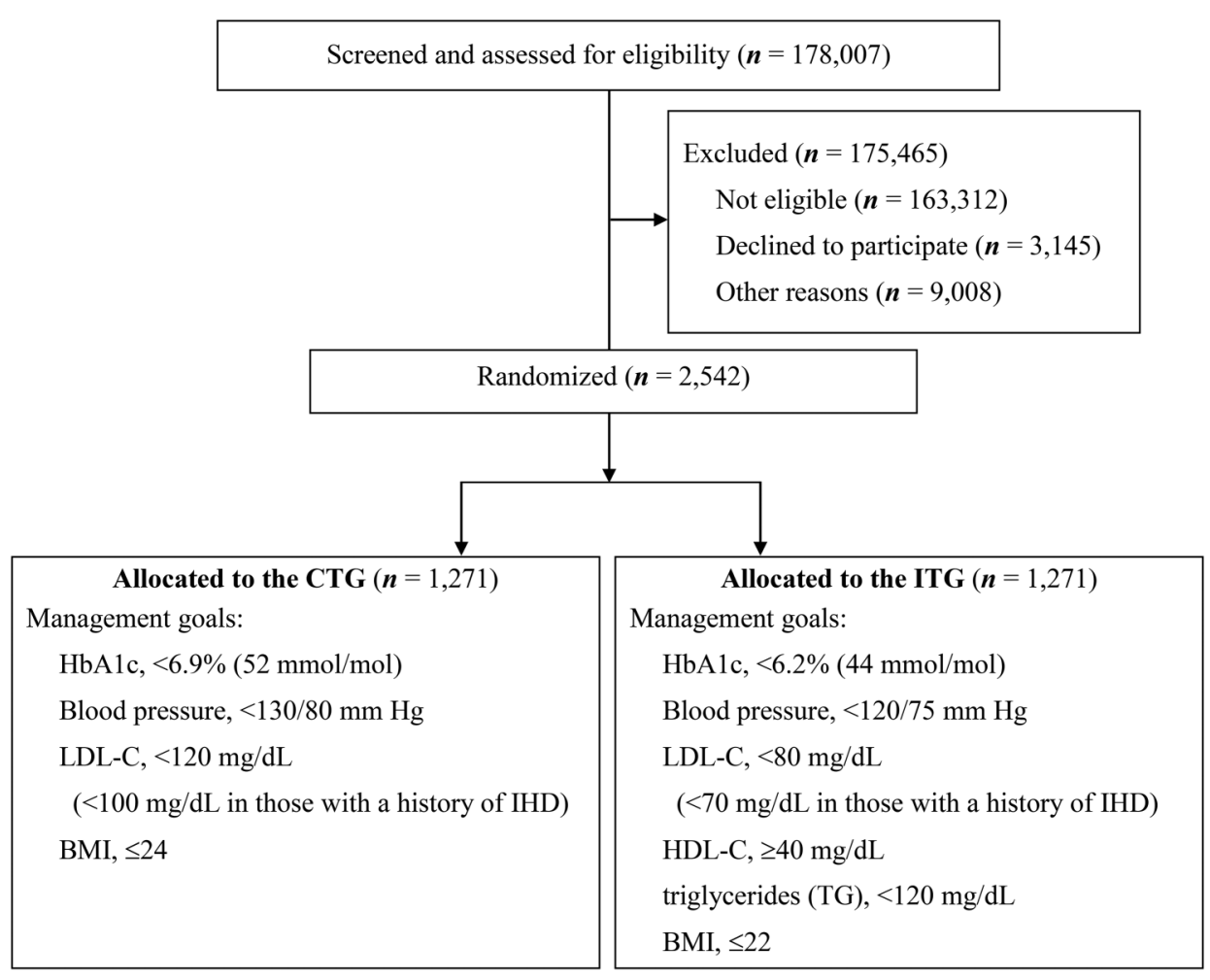


incompetent, consent from a family member or legally authorized representative is received instead.

\section{Management goals and investigational treatment}

The treatment goals were to be set in accordance with the 'Treatment guide for diabetes' edited by the Japan Diabetes Society ${ }^{19}$ in the CTG, and set stricter in the ITG (figure 1), with rationale as follows. The target HbA1c was the value of 'excellently controlled' in the Treatment Guide (2006), which was defined on the basis of the distribution of the HbA1c of the individuals with normal glucose tolerance. The target $\mathrm{BP}$ and low-density lipoprotein cholesterol were set by reference to clinical evidence available when this study was planned, including a meta-analysis on BP lowering by the PSC (Prospective Studies Collaboration), ${ }^{20}$ and results of the CARDS (Collaborative Atorvastatin Diabetes Study), an interventional trial of lipid lowering. ${ }^{21}$ To achieve these target goals, which have been kept unchanged throughout the course of the study, medications in the ITG are intensified in a stepwise manner in 3-6 months, mainly utilizing drugs proven to prevent CVDs (see sFigure 2).

In the ITG, for glycemic control, all participants maintain treatment after registration with predetermined diet and exercise therapy, and if they fail to achieve an HbAlc $<6.2 \%(44 \mathrm{mmol} / \mathrm{mol})$ in the first 3 months, the treatment is intensified with antidiabetic drugs categorized into 4 groups, mainly those that improve insulin resistance with less risk of hypoglycemia. Among insulin sensitizers, pioglitazone, the only thiazolidinedione derivative available in Japan, was preferred because it had emerging evidence of CVD prevention, such as the PROactive study, ${ }^{7}$ when this study was planned. It was also because only low-dose biguanides were allowed to be administered until 2010 in Japan. In the 6 months thereafter, the treatment is to be intensified if they fail to achieve an $\mathrm{HbAlc}<6.2 \%$ or $\geq 1 \%$ decrease in HbA1c (see sFigure 2). If any of the participants receiving treatment on an outpatient basis do not achieve the management goals, hospitalization will be recommended to ensure better glycemic control. All participants in the ITG are provided a BG meter for self-monitoring of BG (SMBG), and the physicians in charge remain informed of the measured results.

All participants maintain treatment for BP on registration with predetermined diet and exercise therapy, and if they fail to achieve $\mathrm{BP}<120 / 75 \mathrm{~mm} \mathrm{Hg}$ in the first 3 months, they are given antihypertensive drugs in steps to ensure that the goal is achieved in 3-6 months, as shown in sFigure 2. All the participants in the ITG are also provided a BP manometer at study entry, and the physician in charge remains informed of these measurements. The physician in charge is to keep informed of self-measured BP for decisions regarding dose escalation or replacement of antihypertensive drugs.

All participants maintain lipid control treatment on registration with predetermined diet and exercise therapy, and if they fail to achieve the lipid control goal in the first 3 months, they are given lipid-lowering drugs, as shown in sFigure 2.

In both groups, all participants with a history of CVDs are given antiplatelet and anticoagulant therapy, such as low-dose aspirin and warfarin, in accordance with the guideline. $^{19}$

It is up to the physician in charge to choose which agent in a class (eg, insulin) to administer. The exceptions are pioglitazone and ezetimibe, both of which are the only drugs in their class (thiazolidinedione derivatives, cholesterol absorption inhibitors, respectively) available in Japan, and so was eicosapentaenoic acid (EPA), which was included in the omega-3 fatty acids thereafter, as is further mentioned later.

\section{Lifestyle modifications}

In both groups, the participants are obliged to follow lifestyle modifications as shown in sTable 3 . Interventions are intensified, focusing on selfmonitoring in daily life, for stricter management goals in the ITG, to improve insulin resistance and reduce body weight. Moreover, the physician in charge remains informed of their measurements and reports on each visit.

The participants in both study groups are instructed on diet by using the "Food exchange lists-dietary guidance for persons with diabetes" edited by the Japan Diabetes Society. ${ }^{22}$ Those with diabetic nephropathy in both study groups are encouraged to restrict protein intake in accordance with the guideline. ${ }^{19}$

\section{Observation and evaluation schedule}

The frequency of hospital visits had been at least once a month, which was modified in January 2010 as follows: (1) at least once a month in those patients receiving insulin therapy and those patients with unstable glycemic control, and (2) a maximum of 3 months apart in those patients who have almost achieved management goals for glycemic control and other factors and whose conditions are stable (except those receiving insulin therapy). An outline of the observation/evaluation schedule for the study is shown in table 1 .

\section{Outcomes}

The end points of the study are shown in table 2, and among them the primary end point and the secondary end point (1) are composite end points. The definitions are set forth, as shown in sTable 4, so that the End Point Assessment Committees could verify each event in an objective fashion.

During the course of the study, general investigations are performed on a regular basis to keep track of the incidence of CVD events as well as to see if the study is progressing as planned.

Even when an event defined as a primary or secondary end point occurred in a particular participant, the participant continues to be part of the study and is observed for occurrence of other events. 


\begin{tabular}{ll}
\hline At regular hospital visits & Body weight, blood pressure (on an outpatient basis) \\
& Blood glucose, HbA1c, lipid panel (total cholesterol, LDL cholesterol (non-HDL \\
& cholesterol), HDL cholesterol, and triglyceride), hematological examination, hepatic/renal \\
& function test, serum electrolytes, and CK \\
& Urinary albumin-creatinine ratio \\
& Body height, waist circumference \\
Every 6 months & Fasting blood glucose, fasting lipid panel \\
Every 12 months & Chest radiograph, echocardiogram, fundus examination \\
& Clinical parameters examined centrally \\
& hs-CRP, fasting serum insulin, and GA \\
& - Fundus examination is also performed within 6 months prior to the end of the study \\
At registration, 1 year later, 3 years & Clinical parameters examined centrally \\
later, and at the end of the study & Adiponectin (including high-molecular-weight adiponectin) \\
& Questionnaires \\
& SF-36, PAID, BDI, PHRF-SCL (SF), and DHQ \\
& - Adiponectin is examined at registration, in March 2013, and at the end of the study \\
At the end of the study & DTSQ and recognition testing \\
\hline
\end{tabular}

$\mathrm{BDI}$, beck depression inventory; DHQ, dietary history questionnaire; DTSQ, diabetes treatment satisfaction questionnaire; GA, glycoalbumin; HbA1c, glycated hemoglobin; HDL, high-density lipoprotein; hs-CRP, high-sensitive $\mathrm{C}$ reactive protein; LDL, low-density lipoprotein; PAID, problem area in diabetes survey; PHRF-SCL (SF), public health research foundation stress check list (short form), SF-36, medical outcomes study-short-form 36-item health survey.

\section{Sample size and study duration}

The trial participants were estimated to include those with ischemic heart disease and those without at a ratio of 3:7, with the occurrence of events, including deaths per year, estimated at $4.4 \%$ (10\% in those with a history of ischemic heart disease and $2 \%$ in those without) in the CTG.

To verify a $30 \%$ risk reduction in the ITG at the twosided significance level of $5 \%$ with a $90 \%$ power, we estimated the required number of events to be 328 , and the required number of patients during the original observation period of 3.75 years was calculated to be 1408 participants per arm by using the Shoenfeld-Richter nomograms.

To recruit sufficient participants, we modified the lower limit of HbAlc in the inclusion criteria from $7.4 \%$

\section{Table 2 Outcomes}

\begin{tabular}{ll}
\hline Primary end & Occurrence of either \\
point & Myocardial infarction \\
& Coronary bypass surgery \\
& Percutangioplasty \\
& Stroke \\
& Carotid endarterectomy \\
& Percutaneous transluminal cerebral \\
& angioplasty \\
Secondary & Carotid artery stenting \\
end points & Death (irrespective of its causes) \\
& 2. Occurrence of myocardial infarction, \\
& 3. Lower or proath \\
& or revascularization of lower limb) \\
& 4. Onset or progression of retinopathy
\end{tabular}

$(57 \mathrm{mmol} / \mathrm{mol})$ to $6.9 \%(52 \mathrm{mmol} / \mathrm{mol})$ in December 2006.

At the end of the accrual period in March 2009, however, the final number of participating participants, 2542 in total (figure 1), and the ratio of those with previous CVD events, $11.0 \%$ (table 3), fell short of the expectation. Thus, we recalculated the required number of events as 250 , by lowering the power to $80 \%$, and the participants are to be followed up until the required events occur to test the hypothesis.

However, the annual event rate was about half of the original estimate, probably due to advances in diabetes care in clinical practice. In January 2010, the primary end point was modified, from 'the incidence of myocardial infarction, stroke, or death', which was thereafter included in the secondary end points, to the present one shown in table 2. Now the study duration is expected to be 9.75 years (until March 2016), yielding a mean intervention duration of approximately 8 years.

\section{Safety}

The physician in charge is to collect data on adverse events, including any of the following four symptoms by using active surveillance: hypoglycemia, edema, palpitation, and shortness of breath. Among them, occurrence of hypoglycemia is to be judged by the physician in charge by taking results of SMBG also into account. Severe hypoglycemia is defined as hypoglycemia requiring someone else's assistance and/or admission.

Serious adverse events include severe hypoglycemia as well as those defined in the ethical guidelines, ${ }^{23}$ which are mentioned later.

The Safety Assessment Committee is to review all reported adverse events, and to make recommendations to the research leader with regard to the handling of the cases (including judgment as to whether the study is 
Table 3 Characteristics of the patients at baseline

\begin{tabular}{|c|c|c|c|}
\hline & CTG $(n=1271)$ & ITG (n=1271) & p Value \\
\hline Age (year) & $59.1 \pm 6.3$ & $58.9 \pm 6.4$ & 0.462 \\
\hline Men/women & $790 / 481$ & $784 / 487$ & 0.838 \\
\hline Body weight (kg) & $65.9 \pm 12.0$ & $65.4 \pm 11.9$ & 0.284 \\
\hline Body mass index & $24.9 \pm 3.8$ & $24.8 \pm 3.6$ & 0.342 \\
\hline Cigarette smoking status (\%) & & & 0.011 \\
\hline Current & 21.0 & 25.8 & \\
\hline Former & 32.7 & 29.2 & \\
\hline Never & 46.3 & 45.0 & \\
\hline Previous CVD event (\%) & 10.8 & 11.2 & 0.800 \\
\hline Duration of diabetes (year) & $8.2 \pm 6.9$ & $8.3 \pm 6.9$ & 0.751 \\
\hline $\mathrm{HbA} 1 \mathrm{c}, \%(\mathrm{mmol} / \mathrm{mol})$ & $\begin{array}{l}8.0 \pm 1.1 \\
(64 \pm 12)\end{array}$ & $\begin{array}{l}8.0 \pm 1.1 \\
(64 \pm 12)\end{array}$ & 0.442 \\
\hline Fasting serum glucose, mg/dL (mmol/L) & $\begin{array}{l}158.8 \pm 39.6 \\
(8.81 \pm 2.20)\end{array}$ & $\begin{array}{l}159.6 \pm 41.5 \\
(8.86 \pm 2.30)\end{array}$ & 0.614 \\
\hline Systolic BP (mm Hg) & $134.1 \pm 16.3$ & $133.5 \pm 16.9$ & 0.357 \\
\hline Diastolic BP (mm Hg) & $80.0 \pm 11.1$ & $79.3 \pm 10.9$ & 0.106 \\
\hline LDL-C, mg/dL (mmol/L) & $\begin{array}{l}125.7 \pm 31.8 \\
(3.25 \pm 0.82)\end{array}$ & $\begin{array}{l}125.5 \pm 30.6 \\
(3.25 \pm 0.79)\end{array}$ & 0.908 \\
\hline HDL-C, mg/dL (mmol/L) & $\begin{array}{l}54.5 \pm 14.0 \\
(1.41 \pm 0.36)\end{array}$ & $\begin{array}{l}54.3 \pm 14.9 \\
(1.40 \pm 0.38)\end{array}$ & 0.728 \\
\hline Triglyceride, mg/dL (mmol/L) & $\begin{array}{c}148.0 \pm 98.2 \\
(1.67 \pm 1.11)\end{array}$ & $\begin{array}{l}148.7 \pm 104.4 \\
(1.68 \pm 1.18)\end{array}$ & 0.851 \\
\hline
\end{tabular}

to be continued with the participants, instructions required to be given to the medical institutions of interest, or whether the trial protocol needs to be revised).

When the ACCORD study was discontinued in February 2008, the research leader temporally suspended new registration and intensification of treatment in the ITG for 2 weeks until the Trial Assessment Committee performed interim analysis and confirmed that (1) GVD events had not been increased in the ITG, (2) occurrence of events had not been correlated with HbA1c, and (3) almost no occurrence of severe hypoglycemia had been seen in the ITG. The Safety Assessment Committee also confirmed that severe adverse events had not been increased in the ITG. Moreover, as was recommended by the committee, the occurrence of death is to be monitored on a real-time basis thereafter.

\section{Discontinuance and dropout}

The criteria for treatment discontinuance are the following: occurrence of a serious macrovascular or microvascular disease, occurrence of another serious complicating disease, diagnosis or strong suspicion of type 1 diabetes (including autoantibody positive), difficulties continuing treatment due to adverse events, patient request for discontinuance, patient death, or other cases in which the physician in charge judged continued treatment would be difficult.

Even when the investigational treatment is discontinued, with the participant's consent (for participants who are incompetent, consent from the family member or legally authorized representative), the participant can be followed up on the presence or absence of the primary and secondary end points of the study.

Dropout cases in this study include (1) those who have discontinued investigational treatment without consent of follow-up, and (2) those with whom the study team have lost contact.

\section{Ethical principles}

The current trial is to be conducted in accordance with the Helsinki declarations, as well as the 'Ethical Guidelines for the Conduct of Clinical Studies' (The MHLW Bulletin, Vol. 459, 2004) and related notifications (Healthcare Administrative Bureau, the MHLW, order 1228 001, December 28, 2004), which were integrated into the 'Ethical Guidelines for Medical and Health Research Involving Human Subjects' (the MHLW and the Ministry of Education, Culture, Sports, Science and Technology, December 22, 2014). ${ }^{23}$ All of the investigators and subinvestigators responsible for the trial must comply with the ethical standards stated earlier. The study was registered at ClinicalTrials.gov supported by the United States National Institutes of Health as NCT00300976. ${ }^{24}$

Following approval by the Central Ethics Committee, the protocol of this study and its revisions are to be reviewed and approved by the ethics committee of each 
institution for their feasibility, as well as their ethical and scientific validity.

\section{Data management}

In accordance with the International Conference on Harmonization Guidelines for Good Clinical Practice, all the data are to be collected from the healthcare institutions by using the electronic data capture system managed by the Data Center and in conformity with the study aims only. Utmost care is to be paid in the handling of personal information, and the participants are to be informed beforehand what personal information is to be composed of and how it is to be used.

\section{Statistical analyses}

Descriptive statistics: The event-free survival period as well as the annual percentage of participants remaining event free is to be calculated by the Kaplan-Meier method, and $95 \%$ CIs are to be calculated using Greenwood's formula. Hypothesis testing: A stratified log-rank test adjusting the stratification factors used for dynamic allocation (except for participating institutions) is to be used to verify the hypothesis that event-free survival is longer in the ITG than in the CTG. Effect size estimates: HR (ITG/CTG) for the event as well as $95 \%$ CIs is to be calculated by using the Cox proportional hazards model. The adjustment/stratification factors used for dynamic allocation (except for participating institutions) are to be incorporated into the regression analysis models. Furthermore, any background factors are also to be incorporated, if they are found to potentially affect the estimates.

After analyses of the primary end points, complementary analyses on the secondary end points are to be performed. As part of the safety evaluation, Fisher's exact (probability) method is to be used to compare the percentages of occurrence of adverse events between the CTG and the ITG, and 95\% CIs for the differences are to be detected. The analyses are to be based on the intention-to-treat principle. Details of the approach to be taken are to be described in the Statistical Analysis Plan.

\section{History of modifications of the protocol}

Modifications in the protocol have been approved by the Central Ethics Committee, including those on inclusion criteria, study duration, frequency of hospital visits, primary end point, funding agency, and the ethical guidelines, as stated earlier.

In addition, new drugs have been added to the available medications in the ITG when at least 1-year safety had been confirmed as a class in clinical practice after appearance on the market. In November 2008, ezetimibe was added to the available medication for dyslipidemia, and in January 2011 DPP-4 inhibitors and GLP-1 receptor agonists were added for hyperglycemia. In December 2012, omega-3 fatty acids were substituted for EPA as a medication for dyslipidemia. Most recently,
SGLT2 inhibitors were included in antidiabetic medication in July 2015.

\section{RESULTS}

All over Japan, 81 healthcare facilities were selected, and the study was open for patient accrual between June 2006 and March 2009. Among the 178007 patients with diabetes screened by the facilities, 14695 were considered eligible, and 5687 received an explanation of the study by the physician in charge. Finally 2542 patients were enrolled, with 1271 allocated to the CTG and the other 1271 to the ITG, until the end of the accrual period, March 2009 (figure 1).

The baseline characteristics, as well as baseline medication, showed no significant change between the CTG and the ITG, except for cigarette smoking status, in which the percentage of current smokers was higher in the ITG (table 3 and sTable 5).

\section{DISCUSSION}

The J-DOIT3 Study Group successfully set up a randomized control study to test the hypothesis that multifactorial intervention therapy could be effective for prevention of CVDs in patients with type 2 diabetes in the Japanese population. We put importance on lifestyle modifications, as well as medications, to reduce hypoglycemia and weight gain.

Recently, the results of the EMPA-REG OUTCOME study were reported, ${ }^{14}$ but a further study is needed to determine the efficacy of SGLT2 inhibitors in CVD prevention. Moreover, the impact of SGLT2 inhibitors on this study is considered to be limited, because only a small number of patients will be exposed by the agents for a short period until the expected end of this study, since these became available quite recently and were included in the therapeutic regimen.

We recruited $>2500$ patients with comorbid hypertension and/or dyslipidemia with a mean HbAlc of $8 \%$ $(64 \mathrm{mmol} / \mathrm{mol})$, and with a mean duration of diabetes of over 8 years. J-DOIT3 is a quite unique study not just because it is the first large-scale trial for East Asian patients with type 2 diabetes who have many different characteristics from Caucasians, but because it also strives to achieve strict and safe control for all the risk factors of vascular complications. Thus, J-DOIT3 will provide novel therapeutic strategies to treat patients with type 2 diabetes for prevention of macrovascular and microvascular complications.

\section{Author affiliations}

${ }^{1}$ Department of Diabetes and Metabolic Diseases, Graduate School of Medicine, The University of Tokyo, Tokyo, Japan

${ }^{2}$ Department of Molecular Sciences on Diabetes, Graduate School of Medicine, The University of Tokyo, Tokyo, Japan

${ }^{3}$ Department of Molecular Diabetic Medicine, Diabetes Research Center, National Center for Global Health and Medicine, Tokyo, Japan

${ }^{4}$ Translational Systems Biology and Medicine Initiative (TSBMI), The University of Tokyo, Tokyo, Japan 
${ }^{5}$ Fiore Kenshin Clinic, Tokyo, Japan

${ }^{6}$ Division of Diabetes and Metabolism, Toho University Ohashi Medical Center, Tokyo, Japan

${ }^{7}$ Department of Internal Medicine, National Center for Global Health and Medicine, Kohnodai Hospital, Chiba, Japan

${ }^{8}$ Department of General Internal Medicine, National Cancer Center Hospital, Tokyo, Japan

${ }^{9}$ Department of Diabetes, Endocrinology and Metabolism, Tokyo Medical University, Tokyo, Japan

${ }^{10}$ Department of Endocrinology and Metabolism, Toranomon Hospital, Tokyo, Japan

${ }^{11}$ Division of Rare/Intractable Disease Research, Department of Research Promotion, Japan Agency for Medical Research and Development, Tokyo, Japan

${ }^{12}$ Department of Integrated Science and Engineering for Sustainable Society, Chuo University, Tokyo, Japan

${ }^{13}$ Department of Diabetes Research, National Center for Global Health and Medicine, Tokyo, Japan

Acknowledgements The authors express their heartfelt gratitude to patients, diabetologists, nurses, and healthcare professionals at the 81 participating institutes. They also show their sincere appreciation to the late Dr Toshitsugu Oda and Dr Takashi Wagatsuma, serving president of the JF-PIMRC, the funding agency of this trial until April 2013, and Dr Yasuhiko Iwamoto, serving president of the JDF, the funding agency thereafter. The authors and the participating investigators of the J-DOIT3 Study Group declared duality of interest also to their healthcare institution.

Contributors KU designed the protocol, steered the study, researched the data, and wrote the manuscript. TS steered the study, researched the data, and wrote the manuscript. MK, YuO, SO, HK, MH, AiM, KO, KH, AtM, and KI steered the study and researched the data. YaO, designed the protocol and steered the study as a biostatistician. MN designed the protocol, steered the study, and researched the data. TK designed the protocol, steered the study, researched the data, and reviewed/edited the manuscript, as the guarantor of this work and takes responsibility for the integrity of the data and the accuracy of the data analysis.

Collaborators The J-DOIT3 Study Group.

Funding The Ministry of Health, Labor and Welfare of Japan; Health and Labour Sciences Research Grants. This study was supported by Health and Labour Sciences Research Grants (H17-Tounyoubyou-tou-Senryaku-801 (Strategic Outcomes Research Program for Research on Diabetes and Comprehensive Research on Diabetes/Cardiovascular and Life-Style Related Diseases), H22-Junkanki-tou(Seishuu)-Shitei-018, and H25-Junkanki-tou (Seishuu)-Shitei-020), by the MHLW. It was also supported by corporate donations from Asahi Kasei Pharma Corporation, Astellas Pharma Inc, AstraZeneca K. K., Bayer Yakuhin, Ltd, Bristol-Myers Squibb K. K., Daiichi Sankyo Co, Ltd, Eli Lilly Japan K. K., Kissei Pharmaceutical Co, Ltd, Kowa Pharmaceutical Co, Ltd, Mitsubishi Tanabe Pharma Corporation, MSD K. K., Nippon Boehringer Ingelheim Co, Ltd, Novartis Pharma K. K., Novo Nordisk Pharma Ltd, Ono Pharmaceutical Co, Ltd, Pfizer Japan Inc, Shionogi \& Co, Ltd, Sumitomo Dainippon Pharma Co, Ltd, and Takeda Pharmaceutical Co, Ltd. Materials for SMBG were provided without any charge from Sanwa Kagaku Kenkyusho Co, Ltd.

Competing interests KU reports a funded research department from MSD, Nippon Boehringer Ingelheim, and Novo Nordisk; lecture fees from Astellas, AstraZeneca, Daiichi Sankyo, Eli Lilly, Kissei, Kowa, Kyowa Hakko Kirin Co, Ltd, Mitsubishi Tanabe, MSD, Nippon Boehringer Ingelheim, Novartis, Novo Nordisk, Ono, Sanofi, Sanwa Kagaku, Shionogi, Sumitomo Dainippon, Taisho Toyama Pharmaceutical Co, Ltd, and Takeda; grants and endowments from Astellas, AstraZeneca, Daiichi Sankyo, Eli Lilly, Kowa, Kyowa Hakko Kirin, Mitsubishi Tanabe, MSD, Nippon Boehringer Ingelheim, Novartis, Novo Nordisk, Ono, Sanofi, Sanwa Kagaku, Sumitomo Dainippon, Taisho Toyama, and Takeda. TS reports lecture fees from Kissei, Mitsubishi Tanabe, and Takeda. YO reports lecture fees from Nippon Boehringer Ingelheim, and Takeda. SO reports lecture fees from AstraZeneca, Eli Lilly, and Mitsubishi Tanabe. HK reports lecture fees from Eli Lilly, Mitsubishi Tanabe, Novo Nordisk, and Ono. MH reports lecture fees from AstraZeneca, Kissei, and
Mitsubishi Tanabe. AiM reports lecture fees from Nippon Boehringer Ingelheim. KO reports lecture fees from Abbott Japan Co, Ltd, ASKA Pharmaceutical Co, Ltd, Astellas, AstraZeneca, Daiichi Sankyo, Eli Lilly, Johnson and Johnson K. K., Kao Corporation, Kyowa Hakko Kirin, MSD, Nippon Boehringer Ingelheim, Novo Nordisk, Ono, Sanofi K. K., and Takeda. $\mathrm{KH}$ reports consulting fees from Kissei and Takeda; research support from Novartis. AM reports lecture fees from Nippon Boehringer Ingelheim. YO reports lecture fees from Eli Lilly, Novo Nordisk, Sanofi, and Takeda; consulting fees from Astellas, Chugai Pharmaceutical Co, Ltd, and Kowa; being the chairman of the board of directors, and owning stock in Statcom Co, Ltd. MN reports lecture fees from AbbVie G. K., Astellas, Daiichi Sankyo, Eli Lilly, Kissei, Kowa, Kyowa Hakko Kirin, Meiji Seika Pharma Co, Ltd, Mitsubishi Tanabe, MSD, Novo Nordisk, Sanofi, Ono, Taisho Toyama, and Takeda; grants and endowments from AstraZeneca, Daiichi Sankyo, Kowa, Kyowa Hakko Kirin, Mitsubishi Tanabe, Mochida Pharmaceutical Co, Ltd, Sanwa Kagaku, and Takeda. TK reports funded research departments from MSD, Nippon Boehringer Ingelheim, Novartis, and Novo Nordisk; lecture fees from Astellas, AstraZeneca, Eli Lilly, Kissei, Kowa, Mitsubishi Tanabe, MSD, Nippon Boehringer Ingelheim, Novo Nordisk, Ono, Sumitomo Dainippon, and Takeda; manuscript fees from Eli Lilly; grants and endowments from Daiichi Sankyo, Mitsubishi Tanabe, Sumitomo Dainippon, and Takeda; funds for contracted research from Daiichi Sankyo, Sanwa Kagaku, and Takeda; funds for collaborative research from Daiichi Sankyo and Novartis.

Patient consent Obtained.

Ethics approval The Central Ethics Committee.

Provenance and peer review Not commissioned; externally peer reviewed.

Data sharing statement No additional data are available.

Open Access This is an Open Access article distributed in accordance with the Creative Commons Attribution Non Commercial (CC BY-NC 4.0) license, which permits others to distribute, remix, adapt, build upon this work noncommercially, and license their derivative works on different terms, provided the original work is properly cited and the use is non-commercial. See: http:// creativecommons.org/licenses/by-nc/4.0/

\section{REFERENCES}

1. Fujishima M, Kiyohara $\mathrm{Y}$, Kato I, et al. Diabetes and cardiovascular disease in a prospective population survey in Japan: the Hisayama study. Diabetes 1996;45(Suppl 3):S14-16.

2. Sone H, Tanaka S, Tanaka S, et al, Japan Diabetes Complications Study Group. Serum level of triglycerides is a potent risk factor comparable to LDL cholesterol for coronary heart disease in Japanese patients with type 2 diabetes: subanalysis of the Japan Diabetes Complications Study (JDCS). J Clin Endocrinol Metab 2011;96:3448-56.

3. Ohkubo Y, Kishikawa H, Araki E, et al. Intensive insulin therapy prevents the progression of diabetic microvascular complications in Japanese patients with non-insulin-dependent diabetes mellitus: a randomized prospective 6-year study. Diabetes Res Clin Pract 1995;28:103-17.

4. [No authors listed]. Intensive blood-glucose control with sulphonylureas or insulin compared with conventional treatment and risk of complications in patients with type 2 diabetes (UKPDS 33). UK Prospective Diabetes Study (UKPDS) Group. Lancet 1998;352:837-53.

5. Stratton IM, Adler Al, Neil HA, et al. Association of glycaemia with macrovascular and microvascular complications of type 2 diabetes (UKPDS 35): prospective observational study. BMJ 2000;321:405-12.

6. Holman RR, Paul SK, Bethel MA, et al. Long-term follow-up after tight control of blood pressure in type 2 diabetes. $N$ Engl $J$ Med 2008:359:1565-76.

7. Dormandy JA, Charbonnel B, Eckland DJ, et al. Secondary prevention of macrovascular events in patients with type 2 diabetes in the PROactive study (PROspective pioglitAzone Clinical Trial In macroVascular Events): a randomised controlled trial. Lancet 2005;366:1279-89.

8. Gerstein HC, Miller ME, Byington RP, et al, Action to Control Cardiovascular Risk in Diabetes Study Group. Effects of intensive glucose lowering in type 2 diabetes. N Engl J Med 2008;358:2545-59. 
9. Patel A, Macmahon S, Chalmers J, et al, ADVANCE Collaborative Group. Intensive blood glucose control and vascular outcomes in patients with type 2 diabetes. N Engl J Med 2008;358:2560-72.

10. Duckworth W, Abraira C, Moritz T, et al. Glucose control and vascular complications in veterans with type 2 diabetes. $N$ Engl $J$ Med 2009;360:129-39.

11. Gaede P, Vedel P, Larsen N, et al. Multifactorial intervention and cardiovascular disease in patients with type 2 diabetes. $N$ Engl $J$ Med 2003;348:383-93.

12. Gaede $\mathrm{P}$, Lund-Andersen $\mathrm{H}$, Parving $\mathrm{HH}$, et al. Effect of a multifactorial intervention on mortality in type 2 diabetes. $N$ Engl $J$ Med 2008;358:580-91.

13. Griffin SJ, Borch-Johnsen K, Davies MJ, et al. Effect of early intensive multifactorial therapy on 5-year cardiovascular outcomes in individuals with type 2 diabetes detected by screening (ADDITION-Europe): a cluster-randomised trial. Lancet 2011;378:156-67.

14. Zinman B, Wanner C, Lachin JM, et al, EMPA-REG OUTCOME Investigators. Empagliflozin, cardiovascular outcomes, and mortality in type 2 diabetes. N Engl J Med 2015;373:2117-28.

15. Yazaki Y, Kadowaki T. Combating diabetes and obesity in Japan. Nat Med 2006;12:73-4.

16. Sakane N, Kotani K, Takahashi K, et al. Japan Diabetes Outcome Intervention Trial-1 (J-DOIT1), a nationwide cluster randomized trial of type 2 diabetes prevention by telephone-delivered lifestyle support for high-risk subjects detected at health checkups: rationale, design, and recruitment. BMC Public Health 2013;13:81.

17. Izumi K, Hayashino Y, Yamazaki K, et al. Multifaceted intervention to promote the regular visiting of patients with diabetes to primary care physicians: rationale, design and conduct of a cluster-randomized controlled trial. The Japan Diabetes Outcome Intervention Trial-2 study protocol. Diabetol Int 2010;1:83-9.

18. The J-DOIT3 Study Group. What is J-DOIT3? http://www.jdoit3.jp/ en/.about.html

19. The Japan Diabetes Society. Treatment guide for diabetes. http:// www.jds.or.jp/common/fckeditor/editor/filemanager/connectors/php/ transfer.php?file=/uid000025_54726561746D656E745F477569646 55F666F725F44696162657465735F323031322D323031332E70 6466

20. Lewington S, Clarke R, Qizilbash N, et al, Prospective Studies Collaboration. Age-specific relevance of usual blood pressure to vascular mortality: a meta-analysis of individual data for one million adults in 61 prospective studies. Lancet 2002;360:1903-13.

21. Colhoun HM, Betteridge DJ, Durrington PN, et al, CARDS investigators. Primary prevention of cardiovascular disease with atorvastatin in type 2 diabetes in the Collaborative Atorvastatin Diabetes Study (CARDS): multicentre randomised placebo-controlled trial. Lancet 2004;364:685-96.

22. The Japan Diabetes Society. Food exchange lists-dietary guidance for persons with diabetes (in Japanese). http://www.jds.or.jp/ modules/en/index.php?content_id=30

23. The Ministry of Health, Labor and Welfare and the Ministry of Education, Culture, Sports, Science and Technology. Ethical guidelines for medical and health research involving human subjects. http://www.lifescience.mext.go.jp/files/pdf/n1500_01.pdf

24. Japan Diabetes Optimal Integrated Treatment study for 3 major risk factors of cardiovascular diseases (J-DOIT3). https://clinicaltrials. gov/ct2/show/NCT00300976 


\section{Correction}

Ueki K, Sasako T, Kato M, et al. Design of and rationale for the Japan Diabetes Optimal Integrated Treatment study for 3 major risk factors of cardiovascular diseases (J-DOIT3): a multicenter, open-label, randomized, parallel-group trial. BMJ Open Diabetes Research and Care 2016;4:e000123. doi:10.1136/bmjdrc-2015000123corr1

The contributors statement has been updated to read: $\mathrm{YaO}$ designed the protocol and steered the study as a biostatistician.

The competing interests statement has been updated to read: $\mathrm{YuO}$ reports lecture fees from Nippon Boehringer Ingelheim, and Takeda. YaO reports lecture fees from Eli Lilly, Novo Nordisk, Sanofi, and Takeda; consulting fees from Astellas, Chugai Pharmaceutical Co, Ltd, and Kowa; being the chairman of the board of directors, and owning stock in Statcom Co, Ltd. MN reports lecture fees from AbbVie G. K., Astellas, Daiichi Sankyo, Eli Lilly, Kissei, Kowa, Kyowa Hakko Kirin, Meiji Seika Pharma Co, Ltd, Mitsubishi Tanabe, MSD, Novo Nordisk, Sanofi, Ono, Taisho Toyama, and Takeda; grants and endowments from AstraZeneca, Daiichi Sankyo, Kowa, Kyowa Hakko Kirin, Mitsubishi Tanabe, Mochida Pharmaceutical Co, Ltd, Sanwa Kagaku, and Takeda.

Affiliation number 14 has also been added for Mitsuhiko Noda. Affiliation 14: Department of Endocrinology and Diabetes, Saitama Medical University, Saitama, Japan.

BMJ Open Diabetes Research and Care 2016;4:e000123. doi:10.1136/bmjdrc-2015-000123corr1 